\title{
Semiotik
}

Band $36 \cdot$ Heft 3-4 (2014)

Seite $89-111$

Stauffenburg Verlag Tübingen

\section{Robert Brandoms Inferentialismus und das Problem der Kommunikation}

\author{
Bernd Prien, Westfälische Wilhelms-Universität Münster
}

\begin{abstract}
Summary. It is generally acknowledged that proponents of a holistic inferentialism face the question of how linguistic communication is possible because their thesis seems to commit them to the view that claims have different meanings for different speakers. In his book Making It Explicit (MIE), Brandom defends a holistic inferentialism and he also concedes that his theory faces the problem of communication just mentioned. In the first part of my contribution, I will offer an account of how exactly this problem arises in the context of Brandom's theory of propositional content. Following that, I will reconstruct Brandom's answer to this problem in part two of my paper. With regard to this answer, however, Daniel Whiting has argued in his article Meaning Holism and De Re Ascription that it contains a gap. In the third part of my paper, I will briefly discuss the nature of the gap in Brandom's answer and propose a way in which this gap could be filled within the theoretical context of MIE.
\end{abstract}

Zusammenfassung. Es ist allgemein anerkannt, dass sich für Vertreter des holistischen Inferentialismus die Frage stellt, wie sprachliche Kommunikation möglich ist. Diese These führt nämlich anscheinend unausweichlich dazu, dass Behauptungen für verschiedene Sprecher verschiedene Bedeutungen haben. In seinem Buch Expressive Vernunft (EV) vertritt Brandom einen solchen Inferentialismus und gibt auch zu, dass sich das Problem der Kommunikation für seine Theorie stellt. Im ersten Teil meines Beitrags möchte ich genauer erläutern, wie sich dieses Problem im Rahmen von Brandoms Theorie propositionalen Gehalts darstellt, bevor ich im zweiten Teil Brandoms Antwort darauf vorstelle. Bezüglich dieser Antwort hat allerdings Daniel Whiting in seinem Aufsatz Meaning Holism and De Re Ascription gezeigt, dass sie eine Lücke aufweist. Im dritten Teil dieses Aufsatzes stelle ich kurz dar, worin diese Lücke besteht, und schlage eine Ergänzung zu Brandoms Antwort vor, die man im theoretischen Rahmen von EV vornehmen könnte. 


\section{Einleitung}

Es ist allgemein anerkannt, dass sich für Vertreter des holistischen Inferentialismus die Frage stellt, wie sprachliche Kommunikation möglich ist (Fodor und Lepore 1992: 8ff.). Dabei ist mit Inferentialismus die These gemeint, dass Behauptungen aufgrund dessen einen bestimmten propositionalen Gehalt haben, dass sie in inferentiellen Beziehungen stehen. Ein Inferentialismus ist holistisch, wenn er davon ausgeht, dass alle Inferenzen, in die eine Behauptung als Prämisse oder Konklusion eingeht, für deren Gehalt relevant sind. Dieser These zufolge kann man insbesondere keine Trennung ziehen zwischen analytischen Inferenzen, die den Gehalt konstituieren, und synthetis ch e n Inferenzen, deren Gültigkeit bloß empirisch ist.

Im Rahmen einer solchen Theorie propositionalen Gehalts entsteht das Problem, dass erfolgreiche Kommunikation unmöglich zu sein scheint. Denn verschiedene Sprecher werden in empirischen Fragen manchmal verschiedene Ansichten haben. Diese drücken sich in der Akzeptanz von Inferenzen aus, die man zwar intuitiv gesprochen als synthetisch bezeichnen würde, die man aufgrund des Holismus aber nicht als für den Gehalt irrelevant ausschließen kann (siehe Abschnitt 2.2). Der Umstand, dass Sprecher bezüglich jeder beliebigen Behauptung zumindest leicht unterschiedliche Mengen von Inferenzen akzeptieren, hat wiederum zur Folge, dass Behauptungen für verschiedene Sprecher unterschiedliche Bedeutung haben. Hieraus wiederum folgt, dass in Unterhaltungen der Sprecher und der Hörer immer ein leicht unterschiedliches Verständnis dessen haben, was gesagt wird. Sie reden also gewissermaßen immer aneinander vorbei (siehe Abschnitt 2.3).

In seinem Buch Expressive Vernunft vertritt Brandom einen holistischen Inferentialismus und gibt auch zu, dass sich das skizzierte Problem der Kommunikation für seine Theorie stellt. Im ersten Teil meines Beitrags möchte ich genauer erläutern, wie sich das Problem im Rahmen von Brandoms Theorie propositionalen Gehalts darstellt, bevor ich im zweiten Teil seine Antwort auf dieses Problem vorstelle. ${ }^{2}$ Bezüglich dieser Antwort hat allerdings Daniel Whiting in seinem Aufsatz Meaning Holism and De Re Ascription gezeigt, dass sie eine Lücke aufweist. Im dritten Teil dieses Aufsatzes stelle ich kurz dar, worin diese Lücke besteht, und schlage eine Ergänzung von Brandoms Antwort vor, die man im theoretischen Rahmen von EV vornehmen könnte.

\section{Holistischer Inferentialismus und das Problem der Kommunikation}

Ich möchte im ersten Teil meines Aufsatzes genauer erläutern, wie sich im Rahmen der von Brandom vertretenen Theorie des Gehalts ein Problem für die Möglichkeit sprachlicher Kommunikation ergibt. Dazu werde ich zunächst seine grundsätzliche Unterscheidung zwischen dem Gebrauch der Sprache und dem Gehalt, der durch diesen Gebrauch instituiert wird, einführen (2.1). Den Gebrauch der Sprache beschreibt Brandom in Form eines besonderen Sprachspiels, dem Spiel des Gebens und Forderns von Gründen (2.2). Die Art, in der Brandom 
dieses Spiel beschreibt, stellt, wie er selbst einräumt, die Möglichkeit der Kommunikation zwischen verschiedenen Sprechern in Frage (2.3). Bevor ich im zweiten Teil meines Aufsatzes erläutere, wie Brandom die Möglichkeit der Kommunikation erklärt, möchte ich im abschließenden Abschnitt (2.4) eine alternative Auffassung des Spiels des Gebens und Forderns von Gründen betrachten, in der sich das Problem der Kommunikation nicht stellt, und erläutern, warum Brandom diese Auffassung zurückweist.

\subsection{Brandoms Unterscheidung zwischen Gebrauch und Gehalt von Behauptungen}

Wie eingangs erwähnt, ist Brandom Inferentialist, was bedeutet, dass er den Begriff des Gehalts mit Rückgriff auf inferentielle Beziehungen erläutert. Propositionaler Gehalt zeichnet sich also wesentlich durch inferentielle Artikulation aus. Diese These steht im Gegensatz zum Repräsentationalismus, demzufolge Gehalt auf der Repräsentation von Gegenständen beziehungsweise Mengen von Gegenständen beruht. Neben dem Inferentialismus besteht eine weitere Grundannahme Brandoms im methodologischen Pragmatismus. Dies bedeutet, dass er die semantischen Eigenschaften von Ausdrücken durch ihre pragmatischen erklären will. Etwas weniger idiomatisch gesprochen, will Brandom den Umstand, dass Ausdrücke eine gewisse Bedeutung haben, dadurch erklären, dass sie in bestimmter Weise gebraucht werden. Um auf diese beiden Aspekte von Ausdrücken zu verweisen, verwendet Brandom die Ausdrücke Semantik und Pragmatik (2002b: 41). ${ }^{3}$

Das Verhältnis zwischen der semantischen Interpretation von sprachlichen Ausdrücken und der pragmatischen Beschreibung ihres Gebrauchs ist nach Brandom analog zu dem Verhältnis zwischen theoretischem und Beobachtungsvokabular. "Methodological pragmatism might usefully be compared with the principle that the point of postulating theoretical objects is to explain the behavior of observable ones" (Brandom 2002b: 43, siehe auch EV: 822). Wir schreiben Ausdrücken also semantische Eigenschaften zu, um ihren Gebrauch erklären zu können; dabei ist eine Zuschreibung aufgrund dessen gerechtfertigt, dass sie und nur sie es erlaubt, den Gebrauch zu erklären.

Dies bedeutet, dass Brandoms Slogan „Die Semantik muss auf die Pragmatik antworten" (EV: 143, Überschrift von Abschnitt 2.2.3) zwei Seiten hat: Obwohl er in erster Linie davor warnen soll, semantische Eigenschaften zu leichtfertig zuzuweisen, sollten methodologische Pragmatisten auch darauf achten, dass die Semantik stark genug ist, alle Aspekte der Pragmatik zu erklären. Auf der einen Seite müssen wir also sicherstellen, dass die semantischen Eigenschaften, die wir zuweisen, not we ndig sind, um den Gebrauch der Ausdrücke zu erklären, auf der anderen Seite sollten diese Eigenschaften aber auch $\mathrm{h}$ i $\mathrm{n}$ re ichend dafür sein.

Die These des methodologischen Pragmatismus sollte man klar von der des Inferentialismus trennen. Inferentialismus besagt, dass unter den semantischen Begriffen derjenige der Inferenz basal ist, und nicht etwa der Begriff der Reprä- 
sentation. Methodologischer Pragmatismus besagt, dass die Zuschreibung semantischer Eigenschaften aufgrund des Gebrauchs gerechtfertigt sein muss. Dies ist grundsätzlich auch mit einem Repräsentationalismus verträglich, denn man kann die These vertreten, dass der semantische Begriff, der als erster unmittelbar aufgrund des Gebrauchs zugeschrieben werden kann, der der Repräsentation ist (MacFarlane 2010: 81ff.). Aber auch wenn dies eine mögliche Position ist, kann man meines Erachtens sagen, dass die Paarung des Inferentialismus mit dem Pragmatismus näher liegt.

Nach dieser allgemeinen Vorbemerkung zum Pragmatismus und seinem Verhältnis zum Inferentialismus möchte ich mich nun Brandoms Konzeption des Gebrauchs von Behauptungen zuwenden, also der diskursiven Praxis, die Gehalte überträgt. Für das Verständnis dieser Konzeption ist meines Erachtens hilfreich, innerhalb ihrer zwei Ebenen zu unterscheiden. Auf der höheren Ebene sind objektiv gültige Normen angesiedelt, die bestimmen, welche inferentiellen Beziehungen zwischen Behauptungen bestehen. Dass diese Normen objektive Gültigkeit haben, bedeutet, dass sie durch die Beschaffenheit der Welt festgelegt sind. Es ist nach Brandom also nicht die Sprachgemeinschaft, die festlegt, welche inferentiellen Beziehungen gelten, sondern die Welt. Diese objektiv gültigen inferentiellen Normen bilden die pragmatischen Eigenschaften von Ausdrücken, durch die die semantische Eigenschaft, einen bestimmten Gehalt zu haben, übertragen wird.

Das Bestehen objektiv gültiger Normen ist allerdings sehr erklärungsbedürftig. Denn erstens ist generell das Bestehen von Normen für naturalistisch orientierte Philosophen wie Brandom erklärungsbedürftig, weil sie nicht Teil der physikalischen Welt sind. Bei objektiv gültigen Normen kommt allerdings noch hinzu, dass sie die Ratifikation durch die Gesamtheit der Sprecher transzendieren. Deren objektive Gültigkeit hat nämlich die Konsequenz, dass es korrekt sein kann, $q$ aus $p$ zu folgern, obwohl kein Sprecher der Sprachgemeinschaft diesen Schluss jemals akzeptiert hat. Deshalb können inferentielle Normen nach Brandom auch nicht dadurch instituiert werden, dass die Mitglieder einer Gemeinschaft bezüglich dieser Inferenz übereinstimmen und sie gemeinsam akzeptieren.

\subsection{Das Spiel des Gebens und Forderns von Gründen}

Eines der Hauptanliegen von EV ist es nun, die Institution objektiver inferentieller Normen zu erklären. Dazu nimmt Brandom eine tiefer liegende Ebene von Praktiken an, die er in Form des Spiels des Gebens und Forderns von Gründen beschreibt. Hierbei handelt es sich, grob gesprochen, um das Spiel des Argumentierens für oder gegen Behauptungen mit dem Ziel herauszufinden, welche von ihnen als wahr akzeptiert werden können. Züge in diesem Spiel bestehen darin, Behauptungen aufzustellen, Folgerungen aus innen zu ziehen und sie zu begründen. Des Weiteren kann man die Behauptungen anderer Sprecher anzweifeln, sie nach den Gründen für ihre Behauptungen fragen und ihren Behauptungen eigene entgegenstellen. ${ }^{4}$ 
Bei der Beschreibung dieses Spiels kann man wiederum zwei Aspekte unterscheiden, einen materialen und einen formalen. Der materiale Aspekt kann bei verschiedenen Durchführungen des Spiels in verschiedenen Sprachgemeinschaften verschieden sein und sich auch im Verlauf einer Durchführung wandeln. Der formale Aspekt bleibt dagegen immer gleich und beschreibt das Spiel als solches.

Der materiale Aspekt des Spiels des Gebens und Forderns von Gründen besteht darin, dass die Sprecher bestimmte inferentielle Beziehungen zwischen Behauptungen anerkennen. Hierbei unterscheidet Brandom zwischen drei Arten von inferentiellen Beziehungen, nämlich den festlegungserhaltenden und den berechtigungserhaltenden Inferenzen sowie den Inkompatibilitätsbeziehungen. Die Sprecher erkennen also erstens an, dass das Aufstellen einer Behauptung sie auf bestimmte weitere Behauptungen festlegt, sie erkennen zweitens an, dass eine Behauptung durch bestimmte andere begründet werden kann, und sie erkennen drittens an, dass die Festlegung auf eine Behauptung die Berechtigung zu bestimmten anderen Behauptungen ausschließt. ${ }^{5}$ Weiterhin gehört es zum materialen Aspekt des Spiels, dass Sprecher die Rechtfertigung von Behauptungen aufgrund von Beobachtung anerkennen, sofern sie unter günstigen Wahrnehmungsbedingungen gemacht wurde, und dass sie bestimmte gewissermaßen selbstverständliche Behauptungen als gerechtfertigt by default anerkennen.

Entscheidend für die Diskussion des Problems der Kommunikation ist dabei, dass nach Brandom jeder Sprecher sein eigenes Repertoire von inferentiellen Beziehungen hat, das er akzeptiert, und dass diese Repertoires zumindest leicht voneinander abweichen. Zwar stimmen die inferentiellen Repertoires verschiedener Sprecher im Großen und Ganzen überein, nach Brandom muss man aber davon ausgehen, dass es vereinzelt auch Differenzen gibt. Dies ergibt sich aufgrund von Überlegungen, die an Quine anschließen. ${ }^{6}$

Man muss davon ausgehen, dass verschiedene Sprecher zum Teil verschiedene inferentielle Beziehungen akzeptieren, weil solche Beziehungen, sofern sie zwischen empirischen Behauptungen bestehen, selbst wieder empirische Behauptungen zum Ausdruck bringen. Dies ist intuitiv plausibel im Fall der Inferenz von Hund auf jagt gerne Katzen, da es ja eine empirische Frage ist, ob Hunde tatsächlich gerne Katzen jagen. Weniger plausibel ist dies dagegen im Fall der Inferenz von Hund auf Säugetier. Um sich von der empirischen Relevanz dieser Inferenz zu überzeugen, sollte man sich die Möglichkeit vor Augen führen, dass Wesen entdeckt werden, die sich in vielen Hinsichten wie Hunde verhalten, aber vom Mars aus ferngesteuerte Roboter sind. Wenn solche Wesen entdeckt würden, wäre es nicht unangemessen, darauf zu reagieren, indem man die genannte Inferenz aufgibt. In diesem Fall würde man erkennen, dass es neben den Hunden, die Säugetiere sind, auch Hunde gibt, die Roboter sind. Solche Beispiele zeigen, dass auch Inferenzen, die scheinbar ausschließlich aufgrund der Gehalte der Prämisse und der Konklusion gelten, empirischen Gehalt aufweisen.

Zieht man nun in Betracht, dass auch Inferenzen von der Art Hund auf Säugetier empirischen Gehalt aufweisen, so ist auf der anderen Seite kein Grund 
mehr zu erkennen, warum man Inferenzen von der Art Hund auf jagt gerne Katzen als für den Gehalt dieser Begriffe irrelevant ansehen sollte. Bei Inferenzen der letztgenannten Art muss man nun allerdings davon ausgehen, dass nur manche Sprecher sie akzeptieren, während andere sie ablehnen würden. Die als Beispiel genannte Inferenz Hund auf jagt gerne Katzen beinhaltet nämlich eine empirische Behauptung, die nicht offensichtlich wahr oder falsch ist, und zu der es deshalb verschiedene Meinungen geben wird. Da wir nun solche Inferenzen bei der Betrachtung des Gehalts von Behauptungen nicht einfach als irrelevant ausschließen können, müssen wir auch davon ausgehen, dass verschiedene Sprecher einer Behauptung verschiedene inferentielle Rollen zuweisen und dass dies auch bei der Übertragung von Gehalt zu berücksichtigen ist. ${ }^{7}$

Diese von Quine angeregten Überlegungen führen zu folgendem Ergebnis: Verschiedene Sprecher erkennen bezüglich einer Behauptung leicht unterschiedliche Mengen von Inferenzen an. Zur theoretischen Erfassung dieses Umstands führt Brandom den Begriff der inferentiellen Signifikanz einer Behauptung ein: Diese ist durch die Inferenzen bestimmt, die ein Sprecher bezüglich dieser Behauptung anerkennt. Da dies davon abhängt, welchen Sprecher man betrachtet, ist die inferentielle Signifikanz sprecher-relativ, so dass man genauer von der inferentiellen Signifikanz sprechen muss, die eine Behauptung für einen Sprecher hat.

Außerdem ist es hilfreich, den Begriff des inferentiellen Repertoires eines Sprechers zu verwenden. Dieses besteht aus der Menge aller inferentiellen Beziehungen, die ein Sprecher anerkennt. Außer den Inferenzen gehören zum Repertoire alle Behauptungen, die ein Sprecher versteht, die also eine bestimmte inferentielle Signifikanz für ihn haben, unabhängig davon, ob er diese Behauptungen für wahr hält oder nicht. Das inferentielle Repertoire kann durch ein Netz dargestellt werden, in dem Knotenpunkte für Behauptungen stehen und Verbindungen zwischen den Knoten für inferentielle Beziehungen.

Bevor ich im Abschnitt 2.3 zum Problem der Kommunikation komme, möchte ich noch kurz auf den formalen Aspekt des Spiels des Gebens und Forderns von Gründen eingehen, der dieses Spiel als solches beschreibt. Während der materiale Aspekt (die von Sprechern akzeptierten Inferenzen) bei verschiedenen Durchführungen des Spiels verschieden sein kann, umfasst der formale Aspekt die immer gleich bleibenden Spielregeln.

Diese fundamentalen Normen des Spiels des Gebens und Forderns geben an, wie Sprecher übereinander ein diskursives Konto führen sollen, in dem sie deren Festlegungen auf und Berechtigungen zu Behauptungen notieren. Diese Normen besagen weiterhin, dass ein Sprecher, der eine Behauptung aufgestellt hat, diese auf eine berechtigte Nachfrage hin auch begründen muss. Weiterhin gibt es (zumindest idealerweise) eine Verpflichtung, Uneinigkeiten zwischen Sprechern zu klären: Wenn ein Sprecher eine Behauptung aufstellt, die der Behauptung eines anderen widerspricht, so können die Sprecher diese Uneinigkeit nicht einfach so bestehen lassen wie eine Uneinigkeit darüber, welche Eissorte am besten schmeckt. Sie sollten argumentativ ermitteln, wer von beiden Recht hat. 
Im weiteren Verlauf meines Aufsatzes werde ich noch zwei weitere Normen des Spiels des Gebens und Forderns von Gründen ansprechen, nämlich die Norm der de re-Zuschreibung und die Norm der rationalen Berichtigung. Diese regeln die Praxis des Kritisierens anderer Sprecher bzw. die Modifikation des eigenen inferentiellen Repertoires angesichts widerspenstiger Erfahrung.

\subsection{Das Problem der Kommunikation}

Ich möchte nun den Prozess der sprachlichen Kommunikation betrachten und erläutern, warum dieser zumindest dem Anschein nach scheitern muss. Da der Sprecher und der Hörer leicht voneinander abweichende Dispositionen bezüglich der Akzeptanz von Inferenzen haben, gibt es im inferentiellen Repertoire des Hörers keine Behauptung, die für inn genau dieselbe Signifikanz hat, wie die aufgestellte Behauptung für den Sprecher. Deshalb muss der Hörer in seinem inferentiellen Repertoire eine Behauptung finden, deren Signifikanz derjenigen sehr ähnlich ist, die die Behauptung für den Sprecher hat. Dass sich eine ähnliche Behauptung finden lässt, setzt voraus, dass beide bezüglich des Großteils der von innen anerkannten Inferenzen übereinstimmen. Dies ist aber normalerweise erfüllt, wenn beide Sprecher in derselben Gemeinschaft aufgewachsen sind. Meiner Ansicht nach hat Brandom diesen Prozess im Auge, wenn er schreibt, die Sprecher müssten „eine Möglichkeit, deren unterschiedliche Repertoires [inferentieller] ${ }^{8}$ Festlegungen aufeinander abzubilden“ (EV: 661) finden.

Die inferentielle Signifikanz, zu der der Hörer so gelangt, weicht natürlich leicht von der ab, die die Behauptung für den Sprecher hat. In diesem Sinne kann man sagen, dass der Hörer ein etwas anderes Verständnis der Behauptung hat als der Sprecher. „Gegeben nun die Relativität der inferentiellen Signifikanz einer Behauptung [...] so folgt daraus, daß die inferentielle Signifikanz bei der Kommunikation nicht erhalten bleibt“ (EV: 668). Erfolgreiche Kommunikation scheint unmöglich zu sein, weil die Gesprächspartner, obwohl sie normalerweise dieselben Wörter verwenden, „verschiedene Begriffe gebrauchen, mit ihren Worten verschiedene Bedeutungen verbinden. Doch es gibt gar nicht so viele Wörter" (EV: 815).

Die Kommunikation scheitert also nicht in dem Sinne, dass der Hörer mit der Behauptung des Sprechers überhaupt nichts anzufangen wüsste. Vielmehr kann man davon ausgehen, dass Gesprächspartner, die zur selben Sprachgemeinschaft gehören, sehr ähnliche Signifikanzen in ihren jeweiligen Repertoires haben, so dass sie die beschriebene Abbildung (way of mapping) vornehmen und auf Behauptungen in sinnvoller Weise antworten können.

Man könnte deshalb die Frage stellen, ob man sich nicht mit dieser vielleicht unvollkommenen, aber immerhin doch ganz guten Übermittlung von Behauptungen zufriedengeben kann. Dies scheint auf den ersten Blick eine gangbare Alternative zu sein, erweist sich aber bei näherer Betrachtung als problematisch, weil die Gesprächspartner dann nicht im strengen Sinne übereinstimmen oder sich widersprechen können. Denn angenommen, der Hörer will dem 
Sprecher, der behauptet, dass $p$, zustimmen. Dazu wird er aufgrund der Aussage des Sprechers ebenfalls die Behauptung, dass $p$, aufstellen. Da diese für inn aber eine etwas andere Signifikanz hat, kann es sein, dass seine Behauptung falsch ist, die des Sprechers aber wahr. Analoge Überlegungen zeigen, dass, wenn der Hörer dem Sprecher widerspricht, also die Negation seiner Behauptung behauptet, beide Behauptungen wahr sein können.

\subsection{Brandoms Zurückweisung gemeinschaftlich geteilter Inferenzen}

Da die Annahme, dass Sprecher leicht voneinander divergierende inferentielle Repertoires haben, zu einer so gravierenden Konsequenz wie der Unmöglichkeit führt, anderen im strengen Sinn zu widersprechen oder zuzustimmen, liegt es nahe, diese Annahme von vornherein vermeiden zu wollen. Dazu könnte man vorschlagen davon auszugehen, dass es eine Teilmenge von Inferenzen gibt, die für den Gehalt von Behauptungen konstitutiv sind, und die durch gesellschaftliche Übereinkunft festgelegt sind. Beim Erwerb der Sprache lernen Kinder von den Erwachsenen, dass diese Inferenzen gültig sind. Es wäre dann sichergestellt, dass alle Sprecher diese Inferenzen teilen und somit Behauptungen in verschiedenen Mündern dieselbe Bedeutung haben.

Eine solche Sichtweise lehnt Brandom allerdings ab, weil sie das Bestehen einer Unterscheidung zwischen analytischen und synthetischen Urteilen impliziert. Eine solche Unterscheidung, so glaubt Brandom wiederum Quine folgend, kann aber nicht gezogen werden. Genauer gesagt, glaubt Brandom nicht, dass man unter den Inferenzen, in die eine Behauptung als Prämisse oder als Konklusion eingeht, eine Trennung vornehmen kann zwischen den Inferenzen, die konstitutiv für den Gehalt dieser Behauptung sind, und den Inferenzen, die nur aus empirischen Gründen gelten. Brandom räumt ein, dass eine solche Unterscheidung intuitiv einleuchtend erscheint. Zum Beispiel scheint die Inferenz von a ist ein Hund auf a ist ein Säugetier konstitutiv für den Begriff Hund zu sein, während die Inferenz von a ist ein Hund auf a jagt gerne Katzen hierfür nicht konstitutiv zu sein und nur empirische Gültigkeit zu haben scheint. ${ }^{9}$

Brandom ist allerdings der Ansicht, dass diese Intuition einer kritischen Überprüfung nicht standhält.

„Die Schwierigkeit mit einem solchen Ansatz ist genau die von Quine betonte: Zu sagen, was genau an den Praktiken des Gebrauchs von Ausdrücken es verdient, so charakterisiert zu werden, daß es einige Behauptungen und Inferenzen, die einen Begriff betreffen, als wesentlich für inn gelten, und andere als solche, die lediglich untergeordnete Informationen über das liefern, worauf er anwendbar ist“ (EV: 674, Übersetzung verändert, B.P.).

Es zeigt sich nämlich, dass bezüglich einer Behauptung jede einzelne Inferenz aufgegeben werden kann, ohne dass sich dadurch der Gehalt der Behauptung ändert. Dies gilt auch für solche Inferenzen, die wir intuitiv gesprochen für analytisch halten würden. Solange nur eine einzelne Inferenz verändert oder auf- 
gegeben wird und die übrigen beibehalten werden, kann man schließen, dass der Gehalt sich nicht ändert. Statt einer scharfen Trennung zwischen Inferenzen, die konstitutiv für den Gehalt einer Behauptung sind, und solchen, die nur empirisch gelten und für den Gehalt irrelevant sind, gibt es ein Kontinuum zwischen mehr oder weniger zentralen Inferenzen, von denen aber keine einzelne strikt notwendig für den Gehalt ist.

\section{Brandoms Antwort auf das Problem der Kommunikation}

Wie wir gesehen haben, entsteht das Problem der Kommunikation, weil verschiedene Sprecher bezüglich einer Behauptung leicht voneinander abweichende Mengen von Inferenzen akzeptieren. Dies ist zunächst eine Feststellung über den Gebrauch von Behauptungen. Auf der Basis dieses Gebrauchs kann man Behauptungen allerdings nur sprecherrelative inferentielle Signifikanzen als semantische Eigenschaften zuschreiben, was dazu führt, dass Gesprächspartner unweigerlich aneinander vorbei reden. Um die Möglichkeit der Kommunikation zu sichern, müsste man den Behauptungen als semantische Eigenschaft einen Gehalt zuweisen, der für alle Sprecher gültig ist. Dies ist auch Brandoms Ziel. Er will zeigen, dass seine Theorie „es zulässt, dass die inferentielle Signifikanz von der doxastischen Perspektive abhängt, nicht aber der begriffliche Gehalt, der eine Funktion von den Perspektiven auf die Signifikanz bestimmt" (EV: 881, Übersetzung verändert, B.P.). ${ }^{10}$

Allerdings ist nicht zu sehen, wie die Zuweisung eines geteilten inferentiellen Gehalts auf der Basis des bisher beschriebenen Gebrauchs möglich ist, denn dieser besteht einfach darin, dass verschiedene Sprecher leicht voneinander abweichende Mengen von Inferenzen akzeptieren. Die Zuschreibung eines geteilten Gehalts setzt also voraus, dass es noch weitere, bisher unbeachtete Aspekte des Gebrauchs von Behauptungen gibt. In diesem Sinne möchte ich im Abschnitt 3.1 die intersubjektive Praxis der de re-Zuschreibung von Meinungen interpretieren. Ich werde dann dafür argumentieren, dass es auf der Basis dieser erweiterten Beschreibung des Gebrauchs möglich und auch nötig ist, den Behauptungen geteilte Gehalte als semantische Eigenschaften zuzuweisen (3.2).

\subsection{Die Praxis der de re-Zuschreibung von Meinungen}

Um zu zeigen, dass es außer den jeweiligen inferentiellen Signifikanzen auch einen für alle Sprecher gültigen Gehalt gibt, muss man meines Erachtens die Praxis der de re-Zuschreibung von Meinungen in Betracht ziehen. Bei dieser Praxis handelt es sich letztlich um eine Erweiterung der Praxis des Abbildens inferentieller Repertoires, die eben schon beschrieben wurde. Sie betrifft den Gebrauch, den ein Hörer von einer Behauptung, dass $p$, machen kann, nachdem er die inferentielle Signifikanz, die sie für den Sprecher hat, auf das eigene inferentielle Repertoire abgebildet hat. Und zwar kann der Hörer die 
Sprecherin auf der Basis seines Verständnisses der Behauptung kritisieren. Genauer gesagt, kann der Hörer aus der Behauptung des Sprechers, dass $p$, so wie er sie versteht, den Schluss ziehen, dass $q$, um dann einzuwenden: $D u$ behauptest, dass $p$, das kann aber nicht sein, weil dann auch q der Fall wäre, während wir beide wissen, dass das nicht so ist! Entscheidend ist dabei, dass der Hörer den Sprecher auch und insbesondere dann auf diese Weise kritisieren kann, wenn nur er (der Hörer) den Schluss von $p$ auf $q$ akzeptiert, der Sprecher aber nicht.

Ich habe die Praxis der Kritik durch den Hörer jetzt allgemein für Inferenzen jeder Art beschrieben. Wenn man diese Praxis nun auf Substitutions-Inferenzen bezüglich singulärer Terme einschränkt, ${ }^{11}$ kann man diese Art der Kritik mit Hilfe von Brandoms Konzeption von de re-Zuschreibungen von Meinungen beschreiben (siehe EV: 8.1.2-3). Angenommen der Hörer hält den singulären Term Wittgenstein für koreferentiell mit Autor des Traktats, während der Sprecher dies nicht tut, da er Wittgenstein nur als Autor der Philosophischen Untersuchungen kennt. Wenn der Sprecher behauptet, dass Wittgenstein einen unsystematischen Schreibstil hatte, so geht der Hörer bei der de re - Zuschreibung einer Meinung, also einer Zuschreibung der Form Du behauptest von a, dass es $F$ ist, folgendermaßen vor: Er ersetzt den singulären Term, den der Sprecher verwendet hat, durch einen anderen, den der Hörer, aber nicht unbedingt der Sprecher für koreferentiell hält, also zum Beispiel Wittgenstein durch Autor des Traktats. Den eingesetzten singulären Term exportiert der Hörer dann in den von-Teil der Zuschreibung und gelangt auf diese Weise zu der Zuschreibung: Du behauptest vom Autor des Traktats, dass er einen unsystematischen Schreibstil hatte.

Diese Ersetzung von Wittgenstein durch Autor des Traktats entspricht dem Schluss von Wittgenstein hatte einen unsystematischen Schreibstil auf Der Autor des Traktats hatte einen unsystematischen Schreibstil. Wenn wir weiterhin als beiden bekannt voraussetzen, dass der Traktat sehr systematisch aufgebaut ist, wird auch plausibel, inwiefern der Hörer den Sprecher aufgrund dieser Folgerung, die der Hörer, aber nicht der Sprecher ziehen würde, kritisieren kann.

Die de re-Zuschreibung und das Kritisieren von Sprechern auf dieser Basis sind Aspekte der diskursiven Praxis, die in der obigen Beschreibung noch nicht erwähnt wurden, die aber unmittelbar an die dort genannte Praxis des Abbildens von Repertoires aufeinander anschließt. Es gehört zu den Normen ( S p i elregeIn), die das Spiel des Gebens und Forderns von Gründen bestimmen, dass man als Hörer die Signifikanz, die eine Behauptung für den Sprecher hat, auf eine ähnliche im eigenen Repertoire abbilden kann, dass man auf der Basis dieses eigenen Verständnisses der Behauptung Schlüsse aus ihr ziehen kann und schließlich den Sprecher dafür kritisieren kann, auf diese Konklusion festgelegt zu sein. 


\subsection{Semantische Interpretation der erweiterten Praxis}

Wenn man diesen neuen Aspekt der diskursiven Praxis berücksichtigt, erweist sich eine semantische Interpretation, die Behauptungen nur sprecherrelative inferentielle Signifikanzen zuweist, als ungenügend, und zwar aus dem Grund, dass nach ihr die de re-Zuschreibung von Meinungen und darauf basierende Kritik als irrational angesehen werden müsste. Wenn Behauptungen so interpretiert werden, dass sie verschiedene Bedeutungen in verschiedenen Mündern haben, müsste man es für irrational halten, dass Hörer die Behauptungen von Sprechern auf der Basis dessen kritisieren, wie sie diese selbst verstehen. Denn der Hörer würde bei seiner Kritik gewissermaßen vermischen, was die Behauptung für inn bedeutet und was sie für den Sprecher bedeutet.

Auf der anderen Seite ist diese Form des Kritisierens Teil der diskursiven Praxis und muss deshalb von der semantischen Interpretation als sinnvoll erklärt werden können. Dies ergibt sich aus der positiven Lesart des Slogans „Die Semantik muss auf die Pragmatik antworten“ (EV: 143). Die Annahme semantischer Eigenschaften soll ja, analog zur Annahme theoretischer Entitäten, den Gebrauch erklären beziehungsweise verständlich machen.

Eine in dieser Hinsicht befriedigende semantische Interpretation der miteinander verflochtenen Gebrauchsweisen von Sprechern, die ihre inferentiellen Repertoires aufeinander abbilden und die ihre Behauptungen auf dieser Basis kritisieren, muss davon ausgehen, dass es einen geteilten Gehalt für beide Gesprächspartner gibt, und dass die inferentiellen Signifikanzen Versuche der jeweiligen Sprecher sind, diesen Gehalt zu erfassen. Vor dem Hintergrund dieser Annahme erscheint die beschriebene Art der Kritik durch den Hörer rational.

„Die unterschiedliche inferentielle Signifikanz von Worten im Munde verschiedener Personen [...] sollte nicht so aufgefaßt werden, als könnten die Gesprächspartner einander nicht wirklich verstehen; vielmehr muss der Gehalt, den sie beide erfassen [...], von verschiedenen Standpunkten aus verschieden spezifiziert werden“ (EV: 818).

Aufgrund der Praxis, dass man Sprecher auf der Basis des eigenen eventuell abweichenden Verständnisses ihrer Behauptungen kritisieren kann, muss man den Gebrauch semantisch so interpretieren, dass es neben den inferentiellen Signifikanzen einen für alle Sprecher gültigen inferentiellen Gehalt gibt, und dass die jeweiligen Signifikanzen Annahmen der verschiedenen Sprecher darüber sind, worin dieser Gehalt besteht, also darüber, welche Inferenzen wirklich gültig sind. Insofern kann man die Signifikanzen als verschiedene Spezifikationen des einen Gehalts von verschiedenen Standpunkten aus bezeichnen.

Diese Lesart wird auch durch das folgende Zitat gestützt: Man betrachte den Fall eines einzelnen Sprechers, der eine neue Überzeugung erwirbt, so dass sich die inferentielle Signifikanz von Behauptungen in seinem Mund ändert. Bezüglich eines solchen Falls stellt Brandom die rhetorische Frage: 
„Doch muss diese Änderung der inferentiellen Signifikanz, die verschiedene Behauptungen für mich haben können, so verstanden werden, daß sie auch eine Änderung beim inferentiellen Gehalt, den sie ausdrücken, involviert?“ (EV: 666).

Mit j a muss man diese Frage nur dann beantworten, wenn man darauf besteht, die semantische Interpretation auf der Basis der zu einer bestimmten Zeit akzeptierten Inferenzen vorzunehmen.

Nach der jetzt vorgenommenen Rekonstruktion von Brandoms Position entsteht das Problem der Kommunikation dadurch, dass man nur die isolierten Gebrauchsweisen einzelner Sprecher betrachtet, die darin bestehen, bezüglich Behauptungen bestimmte Inferenzen zu akzeptieren, während man die intersubjektiv verwobenen Aspekte des Gebrauchs ignoriert, die darin bestehen, dass die Gesprächspartner einander auf der Basis ihres je eigenen Verständnis von Behauptungen kritisieren. Betrachtet man nämlich nur die isolierten Gebrauchsweisen, so ist eine semantische Interpretation unausweichlich, nach der Behauptungen für verschiedene Sprecher Verschiedenes bedeuten. Zieht man andererseits auch die intersubjektiv verwobenen Aspekte des Gebrauchs in Betracht, so wird eine semantische Interpretation erforderlich, nach der es einen für alle Sprecher gültigen Gehalt gibt. Damit ist der Ausgangspunkt, der zum Problem der Kommunikation führt, nicht mehr gegeben.

\section{Whitings Kritik an Brandoms Antwort und eine Ergänzung}

Ich habe nun Brandoms Antwort auf das Problem der Kommunikation, soweit sie sich aus den Kapiteln 7 und 8 von EV ergibt, rekonstruiert. Ich möchte mich abschließend einem Aufsatz von Daniel Whiting zuwenden, in dem er meines Erachtens zutreffend argumentiert, dass diese Antwort noch nicht hinreichend ist. Whiting akzeptiert die oben skizzierten Argumente Brandoms (oder ähnliche) für die These, dass es neben den inferentiellen Signifikanzen, die eine Behauptung für verschiedene Sprecher hat, auch einen geteilten Gehalt gibt. Nach Whiting ist dies als Antwort auf die Frage, wie Sprecher trotz differierender inferentieller Signifikanzen kommunizieren können, unzureichend, weil der inferentielle Gehalt in keine bestimmte Beziehung zu den inferentiellen Signifikanzen gesetzt wird.

\footnotetext{
"And even if some conception of interpersonal content were available - as what is common to all the different attributions of significance made to a given utterance of an expression - it would float entirely free of subjects' attitudes, which would be utterly indifferent to it. Hence the problem of communication remains" (Whiting 2008: 590).
}

Was von dem Sprecher zum Hörer übertragen wird, ist ja zunächst nur die inferentielle Signifikanz, die dabei aber gewissermaßen verfälscht wird. Wenn nun lediglich die Existenz eines geteilten Gehalts nachgewiesen wird, dabei aber unklar bleibt, in welchem Verhältnis dieser Gehalt zu den verschiedenen Signifikanzen steht, bleibt auch unklar, inwiefern der Gehalt im Prozess der Kom- 
munikation übertragen wird. Insofern muss man Whiting darin Recht geben, dass Brandoms Antwort das Problem der Kommunikation bestehen lässt.

Whiting glaubt, dass Brandom dieses Problem durch Hinweis auf den semantischen Externalismus lösen kann, der Teil seiner Theorie ist:

"On his view, the content of a claim [...] is determined by objective inferential relations, that is, relations that correspond not to any perspective but to 'how the world is.' Thus, there is an independent, shareable content, of which all practitioners can have firmer or looser grasps. What performances are proper or improper is a matter of how things stand in the world (MIE: 632)" (Whiting 2008: 591).

Semantischer Externalismus ist grob gesprochen die These, dass der Gehalt von Behauptungen nicht nur davon abhängt, wie wir sie verwenden, das heißt welche Inferenzen wir akzeptieren, sondern auch davon, wie die Welt beschaffen ist, in der wir uns bewegen. Whiting erläutert nicht, warum und in welchem Sinne Brandom einen derartigen Externalismus vertritt. Er geht (zu Recht) davon aus, dass Brandom dies tut (EV: 9.2.4 und EV: 9.3.4) und gesteht um des Argumentes Willen zu, dass sich durch einen Verweis auf den Externalismus die Lücke in Brandoms Antwort auf das Problem der Kommunikation schließen ließe. Whiting versucht dann zu zeigen, dass dieser Externalismus inakzeptable Folgen hätte (Whiting 2008: 591).

Die letztgenannte Frage möchte ich hier nicht weiter verfolgen, sondern statt dessen genauer klären, wie Brandom zum semantischen Externalismus kommt und wie die These des Externalismus mit der Schließung der Lücke in Brandoms Antwort auf das Problem der Kommunikation zusammenhängt. Meiner Ansicht nach geht Whitings Verweis auf den Externalismus in die richtige Richtung, trifft allerdings nicht den Kern dessen, worin die Lücke besteht. Im Kern besteht die Lücke ja darin, dass das Verhältnis zwischen Signifikanzen und Gehalten ungeklärt bleibt, dass der Gehalt "would float entirely free of subjects' attitudes, which would be utterly indifferent to it" (Whiting 2008: 590). Es ist nicht unmittelbar klar, warum ein Verweis auf Brandoms Externalismus diese Lücke schließen kann.

In den folgenden Abschnitten werde ich versuchen, die von Whiting offen gelassenen Fragen zu beantworten. Genauer gesagt möchte ich zeigen, dass der Gehalt nicht, wie Whiting es formuliert, frei über den Einstellungen der Subjekte schwebt und die Einstellungen andererseits unbeeinflusst vom Gehalt sind. Statt dessen besteht nach Brandom eine ganz bestimmte Verbindung zwischen dem geteilten Gehalt und den jeweiligen Signifikanzen (4.1). AbschlieBend zeige ich, dass sich unter Rückgriff auf diese Verbindung einerseits die von Whiting aufgewiesene Lücke in Brandoms Antwort auf das Problem der Kommunikation schließen lässt, andererseits aber auch erklären lässt, warum Brandom einen semantischen Externalismus vertritt. Der Umstand, dass und wie in der Kommunikation geteilte Gehalte übertragen werden, und der Umstand, dass diese Gehalte zum Teil durch die Beschaffenheit der Welt bestimmt werden, erweisen sich als zwei Seiten derselben Medaille (4.2). 


\subsection{Die Verbindung zwischen Signifikanzen und Gehalten}

Wie gesagt, bleibt das oben rekonstruierte Argument für die Möglichkeit der Kommunikation unbefriedigend, weil es die Frage offen lässt, wie der inferentielle Gehalt einer Behauptung sich zu den inferentiellen Signifikanzen verhält, die sie für verschiedene Sprecher hat. Ich möchte nun zeigen, wie sich diese Lücke im Rahmen der in EV entwickelten Theorie schließen lässt. Hier stellt Brandom nämlich Überlegungen zum Verhältnis von Signifikanzen und Gehalt an, auch wenn er diese nicht explizit auf das Problem der Kommunikation bezieht. Und zwar stellt Brandom die These auf, dass Sprecher durch die inferentiellen Signifikanzen, die Behauptungen für sie haben, bei bestimmten inferentiellen Gehalten eingehakt sind, dass sie also „bei diesem oder jenem Begriff eingehakt" (EV: 882, Übersetzung verändert, B.P.) sind. Brandom benutzt außerdem die Metapher, dass wir den Gehalt fassen oder greifen, allerdings nicht direkt, sondern an seinen Griffen. In diesem Bild stehen die Griffe des Gehalts für die inferentiellen Signifikanzen, so dass man sagen kann, dass verschiedene Sprecher denselben Gehalt erfassen, allerdings an verschiedenen Griffen (EV: 876).

Um die These von der Einhakung zu verstehen, muss man meines Erachtens die Praxis der rationalen Berichtigung heranziehen, die Brandom am Ende des 5. Kapitels von EV skizziert (EV: 476; siehe auch Brandom 2008: 184f; 2005: 146; 2002a: 225). Nachdem ich diese Praxis beschrieben habe (4.2.1), möchte ich zeigen, dass man aufgrund dieser Praxis sagen kann, dass Sprecher Begriffe auf eine Weise verwenden sollten, die durch den Zustand der Welt bestimmt ist. Darauf aufbauend schlage ich eine Interpretation der These vor, dass Sprecher aufgrund ihrer inferentiellen Einstellungen bei objektiv in der Welt bestehenden Strukturen eingehakt sind (4.2.2).

Bei der Diskussion dieser Frage ist zu beachten, dass Brandom hier nicht in erster Linie ganze Sätze betrachtet, sondern die Begriffe, die in innen auftreten. Entsprechend zu seiner Unterscheidung zwischen inferentiellen Signifikanzen und inferentiellen Gehalten auf der Ebene ganzer Sätze unterscheidet er auf der subsententialen Ebene zwischen Begriffen und unseren E rfas sunge $n$ von ihnen. Analog zu den inferentiellen Gehalten gibt es also „die Normen, denen der richtige Gebrauch unterworfen ist, in welchem die durch Individuen erfaßten Begriffe bestehen" und analog zu den inferentiellen Signifikanzen gibt es „Dispositionen, Begriffe anzuwenden, Inferenzen zu machen und Sprechakte zu vollziehen, in denen das Erfassen eines Begriffs durch den Einzelnen besteht." Auf diese Weise unterscheidet Brandom „zwischen Begriffen und Vorstellungen von Begriffen“ (EV: 881).

\subsubsection{Die Praxis der rationalen Berichtigung}

Für die Praxis der rationalen Berichtigung (Rational Rectification) ist es von Bedeutung, dass Brandom einen zweiseitigen Inferentialismus vertritt (siehe EV: Kap. 2.5). Dies bedeutet, dass er davon ausgeht, dass der Gehalt einer Behauptung bestimmt ist, sowohl durch die Prämissen, aus denen sie folgt 
(Behauptungen, die gewissermaßen stromaufwärts liegen), als auch durch die Behauptungen, die aus ihr folgen (Behauptungen, die stromabwärts liegen). Einseitige Formen des Inferentialismus gehen dagegen davon aus, dass für den Gehalt von Behauptungen entweder nur relevant ist, woraus sie folgen, oder nur, was aus innen folgt.

Geht man nun von der Ebene ganzer Sätze auf die der Begriffe über, so lässt sich dies am Beispiel des Begriffs der Säure folgendermaßen illustrieren: Angenommen, die Berechtigung zur Anwendung von Säure auf eine Flüssigkeit besteht dann, wenn die Flüssigkeit sauer schmeckt. Das Sauer-Schmecken bezeichnet Brandom dann auch als Bedingung der korrekten Anwendung von Säure. Auf der anderen Seite folge aus der Behauptung, dass eine Flüssigkeit eine Säure ist, die Behauptung, dass sie Lackmus-Papier rot färbt. Wenn man den Begriff Säure auf eine Flüssigkeit anwendet, muss man auch den Begriff färbt Lackmus-Papier rot auf sie anwenden. Dies bezeichnet Brandom auch als Konsequenz der Anwendung des Begriffs.

Die Praxis der rationalen Berichtigung kommt nun in der folgenden Situation zum Tragen: Angenommen, es gibt eine Flüssigkeit, die sauer schmeckt, aber Lackmus-Papier nicht rot färbt. Wenn ich diese Flüssigkeit schmecke, erwerbe ich die Berechtigung, den Begriff Säure auf sie anzuwenden. Behaupte ich nun tatsächlich von der Flüssigkeit, dass sie eine Säure ist, so erwerbe ich eine Festlegung auf die Behauptung, dass sie Lackmus-Papier rot färbt. Wenn ich allerdings Lackmus-Papier in die Flüssigkeit tauche, erwerbe ich durch Beobachtung eine Berechtigung zu der Behauptung, dass die Flüssigkeit Lackmus-Papier nicht rot färbt. Auf diese Art können die Bedingungen zusammen mit den Folgen der Anwendung, die ich für den Begriff Säure anerkenne, mich auf inkompatible Festlegungen führen (EV: 476).

Wenn so etwas geschieht, hat die Welt mir gewissermaßen gezeigt, dass mein Begriff der Säure, der durch die genannten Bedingungen und Folgen der Anwendung charakterisiert ist, inadäquat ist. So wie die Welt tatsächlich beschaffen ist, gibt es nämlich sauer schmeckende Flüssigkeiten, die Lackmus-Papier nicht rot färben.

Wenn ich durch die Bedingungen und Folgen der Anwendung eines meiner Begriffe auf inkompatible Festlegungen geführt werde, so bin ich nach Brandom dazu verpflichtet, meine inferentiellen Einstellungen so zu verändern, dass diese Inkompatibilität vermieden wird (EV: 476). Dazu könnte ich im vorliegenden Beispiel die Bedingung der Anwendung des Begriffs Säure verstärken zu „klare sauer schmeckende Flüssigkeit“ (Brandom 2005, §V). Dieser Praxis, die schon in EV beschreiben wird, gibt Brandom später in Between Saying and Doing den Namen "Rational Rectification" (Rationale Berichtigung) (BSD 189ff.).

\subsubsection{Die Einhakung bei Begriffen im Sinne von objektiven Strukturen}

Wie erwähnt, spricht Brandom davon, dass wir „,bei diesem oder jenem Begriff eingehakt (EV: 882, Übersetzung verändert, B.P.) sind. Diese etwas dunkle Bemerkung ist meines Erachtens so zu verstehen, dass unsere Konzeptionen den Begriffen (objektiven Strukturen) entsprechen sollten, und zwar in einem 
ganz bestimmten Sinne, den ich jetzt mit Rückgriff auf die Praxis der rationalen Berichtigung erläutern werde.

Um zu erläutern, in welchem Sinne Sprecher bei Begriffen eingehakt sind, ist es hilfreich, ein Zwillingserden-Beispiel heranzuziehen, dessen Beschreibung allerdings ein wenig von der klassischen durch Putnam abweicht. Stellen wir uns zwei mögliche Welten vor, die abgesehen von einem bestimmten Detail vollkommen gleich sind. Und zwar wollen wir davon ausgehen, dass es in beiden Welten eine Flüssigkeit gibt, die geschmacksneutral, durstlöschend und durchsichtig ist, bei hinreichender Erhitzung verdampft und Feuer löscht. Allerdings nehmen wir bezüglich dieser letzten Eigenschaft an, dass sie auf der Zwillingserde nur unter fast alle n Umständen besteht, während sie auf der Erde unter allen Bedingungen besteht. Genauer gesagt, soll es auf der Zwillingserde, aber nicht auf der Erde, so sein, dass Wasser unter bestimmten außergewöhnlichen Bedingungen Feuer anheizt, statt es zu löschen. ${ }^{12}$

Betrachten wir nun die Sprecher in beiden Welten. Hier nehmen wir an, dass sie genau dieselbe Konzeption von Wasser haben, die durch folgende Bedingungen und Folgen der Anwendung charakterisiert ist: Die Anwendung von Wasser halten sie für berechtigt, wenn eine Flüssigkeit geschmacksneutral, durstlöschend und durchsichtig ist. Als Folgen der Anwendung von Wasser nehmen die Sprecher an, dass es bei Erhitzung verdampft und Feuer löscht. Auf der Zwillingserde gilt dies tatsächlich zwar nur unter fa st a II e n Umständen, wir wollen aber davon ausgehen, dass den Sprechern dies nicht bekannt ist. Wir nehmen an, dass die Sprecher wissenschaftlich auf dem Stand des 17. Jahrhunderts sind und die Umstände, unter denen Wasser Feuer anheizt, nicht natürlich vorkommen und es das Wissen des 20. Jahrhunderts erfordert, diese in einem Experiment künstlich herzustellen. Die Sprecher auf der Zwillingserde gehen also, genau wie die Sprecher auf der Erde, davon aus, dass daraus, dass etwas Wasser ist, ganz allgemein folgt, dass es Feuer löscht.

Als nächstes möchte ich erläutern, warum die Sprecher in beiden Welten bei verschiedenen Begriffen (objektiven Strukturen) eingehakt sind, obwohl die Konzeption, die sie mit dem Wort Wasserverbinden, die gleiche ist. Dies lässt sich unter Rückgriff auf die Praxis der rationalen Berichtigung erläutern. Dieser Praxis zufolge entsteht für Sprecher, die aufgrund einer ihrer Konzeptionen auf inkompatible Festlegungen geführt werden, wie dies im Fall der Konzeption von Säure erläutert wurde, die Verpflichtung, diese Konzeptionen anzupassen. Bei Sprechern auf der Zwillingserde ist es zwar so, dass ihre Konzeption von Wasser sie bisher nicht auf eine derartige Inkompatibilität geführt hat, weil Wasser Feuer eben nur unter Bedingungen anheizt, die nicht natürlich auftreten und die mit den technischen Mitteln des 17. Jahrhunderts auch nicht hergestellt werden können. Allerdings werden die Sprecher potentiell auf eine solche Inkompatibilität treffen. Wenn sie fortfahren, ihre Umwelt zu erforschen und ihre technischen Fähigkeiten auszuweiten, werden sie eines Tages in der Lage sein, in einem Experiment die relevanten Umstände herzustellen, und werden dann beobachten, dass eine durchsichtige, durstlöschende Flüssigkeit, auf die sie daher Wasser anwenden würden, Feuer in manchen Fällen nicht löscht, sondern anheizt. 
Da die Sprecher auf der Zwillingserde potentiell auf eine Inkompatibilität der relevanten Art geführt werden, kann man von ihnen sagen, dass sie potentiell verpflichtet sind, ihre Konzeption von Wasser zu modifizieren. Außerdem bleiben die Sprecher solange potentiell verpflichtet, ihre Konzeptionen von Wasser zu modifizieren, bis diese vollkommen dem Begriff des Wassers (den objektiven kausalen Strukturen, die bezüglich dieses Stoffs bestehen) entsprechen. In diesem Sinne sollten ihre Konzeptionen von Begriffen den objektiven Strukturen in der Welt entsprechen.

Allgemein lässt sich über Sprecher zu beliebigen Zeiten und in beliebigen möglichen Welten sagen, dass ihre Konzeptionen von Begriffen so beschaffen sein sollten, dass sie sie nicht auf Inkompatibilitäten der Art führen sollten, wie sie an den Beispielen der Säure und des Wasser illustriert wurden. Andernfalls sind sie nämlich verpflichtet, diese Konzeptionen zu verändern. Dies heißt aber wiederum, dass die Konzeptionen der Sprecher mit den objektiv bestehenden Strukturen übereinstimmen sollten. In diesem Sinne ist es meines Erachtens zu verstehen, dass Sprecher „bei diesem oder jenem Begriff eingehakt“ sind (EV: 882, Übersetzung verändert, B.P.).

\section{2 Übertragung von Gehalten und semantischer Externalismus}

Im vorigen Abschnitt habe ich erläutert, dass Sprecher aufgrund ihrer Konzeptionen von Begriffen bei objektiven Strukturen der Welt in dem Sinne eingehakt sind, dass ihre Konzeptionen diesen Strukturen entsprechen sollten. Besteht diese Adäquatheit nämlich nicht, so gibt es immer die Möglichkeit, dass Sprecher aufgrund ihrer Konzeptionen auf inkompatible Festlegungen geführt werden. In diesem Fall wären sie wiederum aufgrund der Praxis der rationalen Berichtigung verpflichtet, ihre Konzeptionen der relevanten Begriffe zu ändern.

Diese Überlegungen wurden auf subsententialer Ebene geführt, genauer gesagt, an Begriffen und unseren Konzeptionen von ihnen. Man kann sie jedoch auch auf ganze Sätze übertragen, so dass man dann sagen kann, dass Sprecher aufgrund der inferentiellen Signifikanz, die eine Behauptung für sie hat, bei einem inferentiellen Gehalt eingehakt sind. Die inferentielle Signifikanz von Das ist Wasser ist von den inferentiellen Beziehungen bestimmt, die ein Sprecher akzeptiert. Aufgrund der Praxis der rationalen Berichtigung gibt es daneben inferentielle Beziehungen, die Sprecher bezüglich Das ist Wasser akzeptieren sollten und die durch die tatsächlichen kausalen Eigenschaften einer bestimmten Flüssigkeit bestimmt sind. Diese inferentiellen Beziehungen bestimmen den Gehalt von Das ist Wasser. Löscht die Flüssigkeit unter allen Umständen Feuer, dann ist es korrekt, allein aus der Prämisse Das ist Wasserauf Das löscht Feuer zu schließen. Löscht sie aber nur unter bestimmten Umständen Feuer, dann ist es auch nur korrekt, diesen Schluss zu ziehen, wenn man als Zusatzprämisse annimmt, dass diese Umstände bestehen.

Die so hergestellte Verbindung zwischen Signifikanzen und Gehalten ist nun in zweierlei Hinsicht von Bedeutung: Auf der einen Seite können verschiedene Sprecher, die derselben möglichen Welt angehören, aufgrund verschiede- 
ner Signifikanzen bei demselben inferentiellen Gehalt eingehakt sein. Hierauf beruht mein Vorschlag zur Schließung der Lücke in Brandoms Antwort auf das Problem der Kommunikation (4.2.1). Auf der anderen Seite können verschiedene Sprecher, für die eine Behauptung dieselbe inferentielle Signifikanz hat, die aber verschiedenen möglichen Welten angehören, bei verschiedenen Gehalten eingehakt sein. Dies ist im obigen Gedankenexperiment bei Sprechern des 17. Jahrhunderts auf der Erde beziehungsweise der Zwillingserde bezüglich der Behauptung Dies ist Wasser der Fall. Dies ist eigentlich nur die zweite Seite derselben Medaille und diese führt zu Brandoms semantischen Externalismus (4.2.2).

\subsubsection{Mittelbare Übertragung von Gehalten in der Kommunikation}

Whitings Einwand hatte ich so rekonstruiert, dass Brandoms Antwort auf das Problem der Kommunikation nur zeigt, dass geteilte inferentielle Gehalte existieren, Brandoms Antwort aber ihr Verhältnis zu den inferentiellen Signifikanzen offen lässt. Da im Prozess der Kommunikation aber zunächst nur Signifikanzen fe h l e r h a ft übertragen werden, bleibt deshalb unklar, inwiefern man davon sprechen kann, dass die geteilten Gehalte übertragen werden. Diese Lücke, dass das Verhältnis zwischen den Signifikanzen und dem Gehalt nicht spezifiziert wird, kann nun mit dem Hinweis auf die Einhakung geschlossen werden. Es hat sich jetzt gezeigt, dass die Inferenzen, die verschiedene Sprecher bezüglich einer Behauptung, akzeptieren (die inferentielle Signifikanz), in einem bestimmten Verhältnis zu den Inferenzen stehen, die wirklich gelten und die den inferentiellen Gehalt der Behauptungen ausmachen.

Aufgrund dieser Verbindung zwischen Signifikanzen und Gehalt kann man nun auch erklären, inwiefern Gehalt in der Kommunikation übertragen wird. Es hatte sich ja gezeigt, dass im Prozess der Kommunikation eine inferentielle Signifikanz gewissermaßen fehlerhaft übertragen wird. Die Signifikanz, die die Behauptung für den Hörer hat, unterscheidet sich leicht von derjenigen, die sie für den Sprecher hat. Auf der anderen Seite stimmen die Signifikanzen zum größten Teil überein, das heißt, die große Mehrheit der inferentiellen Beziehung bezüglich der Behauptung wird von beiden Gesprächspartnern akzeptiert. Deshalb kann man davon ausgehen, dass beide Gesprächspartner aufgrund der jeweiligen inferentiellen Signifikanz bei demselben inferentiellen Gehalt eingehakt sind.

In der Kommunikation wird also unmittelbar eine Signifikanz übertragen, die allerdings bei der Übertragung abgewandelt wird. Mittelbar wird dadurch aber auch ein geteilter Gehalt übertragen, weil beide Gesprächspartner aufgrund der Signifikanzen, die die Behauptung für sie hat, beim gleichen inferentiellen Gehalt eingehakt sind. ${ }^{13}$ Aufgrund der mittelbar übertragenen Gehalte kann der Hörer der Sprecherin nun auch im strengen Sinne zustimmen oder ihr widersprechen. Es kann nicht mehr vorkommen, dass der Hörer die Behauptung der Sprecherin übernimmt, diese für ihn aber zum Beispiel wahr und für sie falsch ist. Analoges gilt für das Widersprechen. 


\subsubsection{Brandoms semantischer Externalismus}

Aus der These der Einhakung bei Gehalten aufgrund von Signifikanzen ergibt sich auch Brandoms semantischer Externalismus, also die These, dass der Gehalt unserer Behauptungen nicht ausschließlich durch den Gebrauch, den wir von ihnen machen, bestimmt ist, sondern zum Teil auch durch die Beschaffenheit der Welt. ${ }^{14}$ Der Gebrauch, den ein Sprecher von einer Behauptung macht, äußert sich ja in der inferentiellen Signifikanz, die die Behauptung für ihn hat. Der Gehalt ist dagegen durch die objektiven Strukturen bestimmt, bei denen der Sprecher aufgrund dieser Signifikanz eingehakt ist.

Im obigen Gedankenexperiment haben wir gesehen, dass die Sprecher auf der Erde und auf der Zwillingserde die Behauptung Das ist Wasserauf die gleiche Weise verwenden, so dass sie dieselbe Signifikanz für sie hat. Gleichwohl sind die Sprecher auf der Zwillingserde bei anderen objektiven Strukturen eingehakt als die Sprecher auf der Erde. Aufgrund der Beschaffenheit ihrer Welt führt die Signifikanz von Das ist Wasser die Bewohner der Zwillingserde potentiell auf inkompatible Festlegungen, was wiederum heißt, dass sie diese Behauptung anders verwenden sollten. Insofern hat aufgrund der anderen Beschaffenheit der Umwelt auf der Zwillingserde die Behauptung Das ist Wasser einen anderen Gehalt als auf der Erde. ${ }^{15}$

Es ist eine wichtige Konsequenz des semantischen Externalismus, dass Sprecher die inferentiellen Gehalte ihrer Behauptungen nicht oder nur annäherungsweise kennen: Es besteht eine „Abhängigkeit dessen, was wir meinen, von den tatsächlichen Verhältnissen, gleichgültig, ob wir wissen, wie sie sind“ (EV: 897, siehe auch EV: 876; EV: 475). Anders gesprochen lernen wir die Gehalte unserer Behauptungen immer besser kennen, je mehr Tatsachen wir über die Welt herausfinden. "For finding out how things really are and finding out what really follows from what and what is really incompatible with what are two aspects of one process" (Brandom 2000b: 359).

Aufgrund dieser Konsequenz könnte man gegen die erweiterte Antwort auf das Problem der Kommunikation folgenden Einwand formulieren: Man kann nicht sagen, dass auf die skizzierte Weise inferentielle Gehalte mittelbar übertragen werden, weil die Gehalte den Gesprächspartnern gar nicht bekannt sind. Damit man davon sprechen kann, dass etwas an den Hörer übertragen wird, muss dieses dem Hörer auch vollständig bekannt sein. Die Gesprächspartner kennen aber nur die jeweilige inferentielle Signifikanz, und deshalb kann auch nur von dieser gesagt werden, dass sie (verfälschend) übertragen wird.

Meiner Ansicht nach ist dieses Bedenken durchaus berechtigt, es betrifft jedoch allgemein Brandoms Theorie des Gehalts von Behauptungen, und nicht spezifisch die jetzt rekonstruierte Antwort auf das Problem der Kommunikation. Dieses Bedenken betrifft nämlich eigentlich Brandoms These, dass Sprecher den inferentiellen Gehalt ihrer Behauptungen erfassen, obwohl sie ihn nur fehlerhaft durch die Signifikanzen spezifizieren können, die sie für sie haben. Sprecher wissen zwar nur annäherungsweise, welchen Gehalt Behauptungen haben, trotzdem erfassen sie nach Brandom diesen Gehalt. Sie erfassen ihn nicht direkt, sondern an seinen Griffen, wie Brandom es metaphorisch aus- 
drückt (EV: 876). Wenn man diese Sichtweise des Erfassens von Gehalt akzeptiert, muss man auch zugeben, dass in der Kommunikation Gehalt übertragen wird. Denn dann muss man zugeben, dass der Hörer den Gehalt, an den Griffen (das heißt aufgrund der inferentiellen Signifikanz), die er verwendet, erfasst, obwohl er ihn nur näherungsweise kennt.

\section{Anmerkungen}

$1 \quad$ Im Folgenden abgekürzt als EV; der Titel des englischen Originals lautet Making It Explicit (MIE).

2 Siehe hierzu auch Prien (2010, Abschnitt 1 und 2).

3 An den durch die Praxis übertragenen Gehalt stellt Brandom zwei Forderungen, die zu erfüllen die offizielle Zielsetzung seines Buches ausmachen. Zunächst will Brandom zeigen, dass der übertragene Gehalt eine repräsentationale Dimension aufweist. Wenn man Gehalt so charakterisiert, dass er in inferentiellen Beziehungen besteht, lässt sich ja die Frage stellen, ob Behauptungen sich aufgrund inres Gehalts auch auf Objekte beziehen. Eine weitere Herausforderung ist die objektive Gültigkeit von Inferenzen. Nach Brandom besteht die Gültigkeit von Inferenzen unabhängig davon, ob irgendein Sprecher oder alle Sprecher sie jemals akzeptiert oder zurückgewiesen haben.

4 Bei der Beschreibung des Spiels dürften eigentlich keine semantischen Ausdrücke wie Behauptung oder Inferenz benutzt werden, sondern nur normative wie Festlegung und Berechtigung oder naturalistische wie Disposition. Wenn dies dennoch geschieht, so ist dies als Vorgriff auf die anvisierte semantische Interpretation des Gebrauchs zu verstehen.

5 Dabei ist mit Anerkennung die praktische Anerkennung gemeint, die in einer Disposition besteht, einen Schluss zu ziehen. Zusätzlich zu dieser praktischen Anerkennung kann man eine inferentielle Festlegung auch explizit anerkennen, indem man das Konditional Wenn $p$, dann $q$ als Behauptung aufstellt. Dies ist allerdings nicht vorausgesetzt, wenn hier von Anerkennung von Inferenzen die Rede ist.

6 Siehe Quines Aufsatz Two Dogmas of Empiricism, Abschnitt 6.

7 Brandom präsentiert ein etwas anderes Argument für die These, dass verschiedene Sprecher verschiedene Inferenzen akzeptieren: Was ein Sprecher aus einer Behauptung, dass $p$, folgern würde, hängt davon ab, welche zusätzlichen Prämissen er akzeptiert. Wenn die Inferenz von $p$ auf $q$ von der Zusatzprämisse $r$ abhängt, werden Sprecher, die $r$ akzeptieren, $q$ aus $p$ folgern, während Sprecher, die $r$ nicht akzeptieren, dies nicht tun würden. Da verschiedene Sprecher verschiedene Zusatzprämissen akzeptieren, würden sie aus der Behauptung, dass $p$, auch verschiedene Folgerungen ziehen.

8 Brandom betrachtet an dieser Stelle nicht Inferenzen im Allgemeinen, sondern nur Substitutions-Inferenzen. Deshalb spricht er hier von substitutionalen Festlegungen, die einen Spezialfall der inferentiellen Festlegunge $\mathrm{n}$ bilden. 
9 Diesen Ansatz, den Brandom nicht verfolgt, besteht darin, Inferenzen „ungleich zu behandeln und eine privilegierte Klasse von ihnen als konstitutiv für den Begriff zu betrachten. Den übrigen Inferenzen würde ein Status zweiter Klasse zuerkannt dahingehend, daß sie sich als richtige Weisen herausstellen, den so konstituierten Begriff zu gebrauchen“ (EV: 878).

10 Analog kann man bezüglich eines Sprechers, der zu verschiedenen Zeiten verschiedene Zusatzprämissen akzeptiert und deshalb verschiedene Inferenzen akzeptieren würde, fragen: „Doch muss diese Änderung der inferentiellen Signifikanz, die verschiedene Behauptungen für mich haben können, so verstanden werden, daß sie auch eine Änderung beim inferentiellen Gehalt, den sie ausdrücken, involviert?" (EV: 666).

11 Substitutions-Inferenzen sind Inferenzen, die im grundlegenden Fall eine der beiden folgenden Formen haben: Aus Fa folgt Ga oder aus Fa folgt Fb. Syntaktisch gesehen gelangt man hier von der Prämisse zur Konklusion, indem man entweder das Prädikat oder den singulären Term durch ein anderes/einen anderen ersetzt. Die Rede von Substitutions-Inferenzen setzt also voraus, dass die Prämisse und die Konklusion eine Struktur aus Prädikat und singulärem Term aufweisen (siehe EV: Kap. 6.4). Damit die genannten Substitutions-Inferenzen gültig sind, muss die Extension des Prädikats $G$ die von $F$ umfassen bzw. die singulären Terme $a$ und $b$ den gleichen Gegenstand bezeichnen. Man schließt dann z.B. von Das dort ist eine Eiche auf Das dort ist ein Baum, bzw. von Kolumbus war Spanier auf Der Entdecker Amerikas war Spanier.

12 Um dieses Szenario an die übliche Beschreibung des Unterschieds zwischen Erde und Zwillingserde anzuschließen, wonach Wasser $\mathrm{H}_{2} \mathrm{O}$ bzw. XYZ ist, kann man zusätzlich annehmen, dass die auf der Erde beobachtbaren Eigenschaften am besten durch die atomare Struktur $\mathrm{H}_{2} \mathrm{O}$ erklärt werden, während die auf der Zwillingserde beobachtbaren Eigenschaften am besten durch die atomare Struktur XYZ erklärt werden.

13 Es wird an dieser Stelle deutlich, dass es zum Füllen der von Whiting aufgewiesenen Lücke nicht hinreicht, irgendein Verhältnis zwischen Signifikanzen und Gehalt anzugeben. Vielmehr ist dafür erforderlich, dass ähnliche Signifikanzen zum gleichen Gehalt ins Verhältnis gesetzt werden.

14 Klassischerweise versteht man unter dem semantischen Externalismus die These, dass die Referenz von Termen für natürliche Arten nicht ausschließlich durch die Vorstellungen bestimmt ist, die wir mit innen verbinden, sondern zum Teil auch durch die physikalische Umwelt, in der wir leben. Hilary Putnam, der diese These mit dem Slogan "'meanings' just ain't in the head!" (1985: 227) zusammengefasst hat, gilt gemeinhin als Urheber dieser Sichtweise. Brandoms pragmatistische Fassung des semantischen Externalismus unterscheidet sich von der ursprünglichen Form dadurch, dass er nicht mentale Zustände als ausschließliche Determinante von Bedeutungen zurückweist, sondern den Gebrauch von Sprechakten.

15 Da Brandom gleichzeitig am methodologischen Pragmatismus festhält, also an der These, dass die semantischen Eigenschaften durch die diskursive Praxis festgelegt werden, muss er schließen, dass diese Praxis über das Spiel des Gebens und Forderns von Gründen hinausgeht und auch die Beschaffenheit der Welt, in der die Sprecher leben, mit beinhaltet. Nach Brandom gilt daher: „Welche wohl- 
bestimmten Praktiken eine Gemeinschaft hat, hängt davon ab, wie die Tatsachen sind" (EV: 476).

\section{Literatur}

Brandom, Robert (1994), Making It Explicit. Cambridge, Mass.: Harvard University Press. Brandom, Robert (2000a), „Vocabularies of Pragmatism“. In: Robert Brandom (ed.), Richard Rorty: The Philosopher Meets his Critics. Oxford: Blackwell: 156-183.

Brandom, Robert (2000b), „Facts, Norms, and Normative Facts: A Reply to Habermas“. European Journal of Philosophy 8: 356-374.

Brandom, Robert (2000c), Expressive Vernunft: Begründung, Repräsentation und diskursive Festlegung. Frankfurt a.M.: Suhrkamp.

Brandom, Robert (2002a), Tales of the Mighty Dead. Cambridge, Mass.: Harvard University Press.

Brandom, Robert (2002b), „Pragmatics and Pragmatisms“. In: James Conant und Urszula M. Zeglen (eds.), Hilary Putnam: Pragmatism and Realism. London: Routledge: $40-59$.

Brandom, Robert (2005), „Sketch of a Program for a Critical Reading of Hegel“. In: K. Ameriks und J. Stolzenberg (eds.), International Yearbook of German Idealism 3. Berlin: De Gruyter: 131-161.

Brandom, Robert (2008), Between Saying and Doing. Oxford: Oxford University Press. BSD: Siehe Brandom 2008.

EV: Siehe Brandom 2000c.

Fodor, J. und Lepore, E. (1992), Holism: A Shopper's Guide. Oxford: Blackwell.

MacFarlane, John (2010), „Pragmatism and Inferentialism“. In: Bernhard Weiss und Jeremy Wanderer (eds.), Reading Brandom on Making It Explicit. London: Routledge: 81-95.

MIE: Siehe Brandom 1994.

Putnam, Hilary (1985), „,The Meaning of 'Meaning'“. In: Hilary Putnam (ed.), Philosophical Papers. Vol. 2. Mind, Language and Reality. Cambridge University Press:215271.

Quine, Willard Van Orman (1961), „Two Dogmas of Empiricism“. In: Willard Van Orman Quine (ed.), From a Logical Point of View. New York: Harper \& Row. 2. Auflage: 20-46.

Prien, Bernd (2010), „Robert Brandom on Communication, Reference, and Objectivity“. International Journal of Philosophical Studies 18: 433-458.

Scharp, Kevin (2003), „Communication and Content“. International Journal of Philosophical Studies 11: 43-61.

Whiting, Daniel (2008), „Meaning Holism and De Re Ascription“. Canadian Journal Of Philosophy 38: 575-600. 


\section{PD Bernd Prien}

Westfälische Wilhelms-Universität

Philosophisches Seminar

Domplatz 6

D-48143 Münster

E-Mail: bprien@uni-muenster.de 